\title{
EL ZINC EN LA SALUD HUMANA - I
}

\section{ZINC IN HUMAN HEALTH - I}

\author{
Daniel López de Romaña (1), Carlos Castillo D. (2), Doricela Diazgranados (1)
}

(1) Laboratorio de Micronutrientes, Instituto de Nutrición y Tecnología de los Alimentos, Universidad de Chile, Santiago, Chile.

(2) Departamento de Pediatría y Cirugía Infantil, Campo Centro, Facultad de Medicina, Universidad de Chile, Santiago, Chile.

\begin{abstract}
Zinc is a divalent cation with multiple functions in the human body. Zinc absorption occurs in the small intestine and, together with zinc excretion via feces, are the point of control for zinc homeostasis. Zinc is an essential nutrient with a specific role in more than 300 enzymes which participate in all important biochemical reactions of the human body. Therefore, zinc status has a direct effect in growth, neurological and behavioral development and in the immune system. The diagnosis of zinc deficiency in individuals is difficult given that there is still no indicator with adequate sensitivity. Nevertheless, it is acceptable to use serum zinc to evaluate populations. Finally, intervention strategies such as supplementation, food fortification and/or dietary modification can be used to control and/or prevent zinc deficiency.
\end{abstract}

Key words: zinc; health; homeostasis; deficiency; interventions.

Este trabajo fue recibido el 18 de Diciembre de 2009 y aceptado para ser publicado el 2 de Mayo de 2010.

\section{ZINC COMO NUTRIENTE EN HUMANOS}

La importancia del zinc como un nutriente esencial para la salud humana es de amplio conocimiento. A comienzos de la década de 1930 estudios en animales mostraron la importancia del zinc en el crecimiento y supervivencia de los animales (1). Más adelante, en 1961, Prasad y colaboradores reconocieron la importancia de la deficiencia de zinc en humanos (2). Ahora se conoce que más de 100 enzimas necesitan zinc para su función catalítica. A diferencia de otros elementos traza, se pueden encontrar ejemplos de enzimas que requieren zinc en las seis clases de enzimas (oxidoreductasas, transferasas, hidrolasas, lisasas, isomerasas y ligasas) (3). Por ende, el zinc participa en una gama de procesos bioquímicos relacionados con el metabolismo humano y no es extraño que múltiples funciones fisiológicas y metabólicas se vean alteradas cuando ocurre su deficiencia.

El zinc tiene funciones catalíticas, estructurales y reguladoras. La anhidrasa carbónica, carboxipeptidasas, fosfatasa alcalina y la $\beta$-lactamasa son algunas enzimas en las que el rol catalítico del zinc es necesario para su función biológica. En su rol estructural el zinc estabiliza la estructura terciaria de enzimas, dándoles una forma conocida como "dedos de zinc", las cuales se unen al ADN para la trascripción y expresión génica. Se ha estimado que el $3 \%$ de los genes codifican proteínas que contienen dedos de zinc (4). Ejemplos de factores de transcripción de dedos de zinc son los receptores del ácido retinoico y del 1,25-dihidroxicalciferol (5). Finalmente, los iones de zinc intracelulares cumplen una función reguladora activando o inhibiendo ciertos factores que son responsables de regular expresión genética (6).

El zinc se encuentra presente en todos los órganos, tejidos, fluidos y secreciones del cuerpo humano (7). Aproximadamente el $83 \%$ del zinc en el cuerpo está en músculo y hueso (tabla 1) y el $95 \%$ se encuentra a nivel intracelular. No existe un lugar anatómico específico que funcione como reserva de zinc y por ende no hay reservas convencionales en tejidos que puedan ser liberadas o almacenadas en respuesta a variaciones en la dieta.

El zinc se absorbe a nivel intestinal, mayoritariamente en el duodeno y yeyuno (8). El transporte dentro del enterocito se da mediante un proceso saturable mediado por la proteína Zip4 (9). El zinc también se 
puede absorber pasivamente a nivel paracelular, pero sólo cuando hay una ingesta elevada (10). Se piensa que el zinc es transportado fuera del enterocito hacia la circulación portal mediante la proteína transportadora de zinc-1 (ZnTP-1) (9). La circulación portal lleva el zinc absorbido directamente al hígado, donde es incorporado y rápidamente liberado a la circulación sistémica para ser llevado a otros tejidos. Aproximadamente el $70 \%$ del zinc en circulación esta unido a la albúmina.

El zinc plasmático, usado frecuentemente como indicador del estado de zinc, se encuentra regulado homeostáticamente, de tal modo que individuos con una deficiencia marginal pueden tener valores normales (11). Adicionalmente, el zinc plasmático puede ser influenciado por varios factores no nutricionales, tales como infecciones (disminución por redistribución del zinc desde el plasma hacia el hígado), enfermedades asociadas a una hipoalbuminemia, embarazo (disminución por expansión del volumen sanguíneo), y enfermedades asociadas a hemólisis (aumento por liberación del zinc en eritrocito al plasma) (11). Se sabe que existe un intercambio entre el zinc en plasma y aquel en órganos y tejidos del cuerpo. De esta manera, se observa un intercambio lento entre el zinc en plasma y el zinc en huesos y músculo y un intercambio rápido con el zinc en hígado y eritrocitos $(12,13)$. Se postula que los llamados pools de zinc de intercambio rápido tienen un rol importante en el metabolismo de zinc pues pudieran reflejar una ingesta reciente $(14,15)$.

Las pérdidas de zinc a nivel gastrointestinal, producto de secreciones pancreáticas, biliares e intestinales, corresponden a la mayoría del zinc eliminado del cuerpo (16). Sin embargo, mucho del zinc secretado al intestino es re-absorbido por el organismo (16). Otras vías de excreción de zinc son la orina, sudor, semen y cabello. El intestino juega un rol de suma importancia en el control homeostático del metabolismo de zinc debido a que este control se da mediante la regulación de la absorción y excreción endógena del zinc proveniente de la dieta. Hay una relación indirecta entre la cantidad de zinc ingerido y la absorción fraccional de zinc (proporción de zinc en la dieta que es absorbido) y esto tiene un impacto directo en la cantidad total de zinc que es absorbido (17-19). Por ejemplo, en situaciones donde ocurre una

\section{TABLA 1}

\section{Contenido de zinc en principales órganos y tejidos en un adulto $(70 \mathrm{Kg})^{1}$}

\section{Tejido}

Músculo

Esqueleto

Hueso

Médula

Cartílago

Tejido periarticular

Hígado

Pulmón

Piel

Sangre

Riñon

Cerebro

Dientes

Cabello

Bazo

Nódulos linfáticos

Tracto gastrointestinal

\section{Concentración de zinc}

(mg/kg peso húmedo)

\section{Cantidad total de zinc (mg)}

1400

72

40

39

33

15

14

11,5

4

3,6

3,5

1,8

\section{Porcentaje del total de} zinc en el cuerpo (\%)
63

20

3

1

$<1$

3

2

2

1

1

1

1

$<1$

$<1$

$<1$

$<1$

${ }^{1}$ Adaptado de: International Zinc Nutrition Consultative Group (IZiNCG). Technical Document \#1.

Assessment of the risk of zinc deficiency in populations and options for its control. Food Nutr Bull 2004; 25(suppl 2): S94-204. 
restricción de la ingesta de zinc, se ve un aumento en la absorción fraccional de zinc $(17,18)$, aunque pareciera que la absorción no es una adaptación a largo plazo (15). Aún no esta definido si la absorción fraccional de zinc esta regulada por el estado de zinc de un individuo. Por otro lado, se sabe que la excreción endógena de zinc se regula en respuesta a los cambios en la ingesta de zinc de un individuo. En situaciones donde existe una restricción en la ingesta la excreción endógena disminuye $(15,19)$.

A nivel celular, la regulación de la homeostasis de zinc se da principalmente a nivel del epitelio intestinal y tejido pancreático, orquestada por los transportadores de zinc que se encuentran en estos tejidos. Se ha observado que una restricción dietaria de zinc en ratas estimula la expresión de la proteína Zip4, la cual se moviliza hacia la membrana apical para presumiblemente promover mayor incorporación del zinc dietario al enterocito (9). Por otro lado, se reprime la expresión de la proteína Zip5, la cual normalmente se localiza en la membrana basolateral del enterocito promoviendo el ingreso de zinc desde el plasma, y la proteína es internalizada y degradada (20). Finalmente, se reprime la expresión de la metalotionina (21). Paralelamente, a nivel de tejido pancreático disminuye la expresión de la proteína ZnT1, lo cual reprime la salida de zinc de las células acinares del páncreas para su posterior secreción a nivel intestinal (22) y también se internaliza y degrada la proteína Zip5 (9), previniendo el flujo de zinc desde el plasma hacia las células acinares. Como consecuencia de todas estas acciones se estimula la absorción de zinc y se reprime la excreción endógena a nivel intestinal.

\section{ZINC EN ALIMENTOS Y FACTORES EN LA DIETA QUE AFECTAN SU ABSORCIÓN}

El zinc se encuentra en una variedad de alimentos, pero las mayores concentraciones de zinc se dan en alimentos de origen animal, particularmente en los órganos y músculos de vacunos, porcinos, aves, pescados y mariscos y, en menor medida en huevos y lácteos. El contenido de zinc es relativamente alto en nueces, semillas, legumbres y cereales sin refinar y bajo en tubérculos, cereales refinados, frutas y verduras (tabla 2).

La cantidad total de zinc en la dieta solo provee un estimado crudo de la ingesta de zinc, pues existen muchos factores que afectan su biodisponibilidad (10). Biodisponibilidad se refiere a la proporción de zinc que

\section{TABLA 2}

\section{Contenido de zinc, densidad de zinc, contenido de fitato y relación molar fitato-zinc de alimentos comúnmente consumidos ${ }^{1}$}

\begin{tabular}{lcccc}
$\begin{array}{c}\text { Grupos } \\
\text { de alimentos }\end{array}$ & \multicolumn{2}{c}{ Contenido de Zinc } \\
mg/100 g & $\mathbf{m g} / \mathbf{1 0 0}$ Kcal & $\begin{array}{c}\text { Contenido de fitato } \\
\text { mg/100 g }\end{array}$ & $\begin{array}{c}\text { Relación molar } \\
\text { Fitato:Zinc }\end{array}$ \\
\hline Hígado, riñón & & & 0 & 0 \\
Carne & $4,2-6,1$ & $2,7-3,8$ & 0 & 0 \\
Aves & $2,9-4,7$ & $1,1-2,8$ & 0 & 0 \\
Pescados, mariscos & $1,8-3,0$ & $0,6-1,4$ & 0 & 0 \\
Huevos & $0,5-5,2$ & $0,3-1,7$ & 0 & 0 \\
Lácteos & $1,1-1,4$ & $0,7-0,8$ & 0 & 0 \\
Semillas, nueces & $0,4-3,1$ & $0,3-1,0$ & $1760-4710$ & $22-88$ \\
Menestras & $2,9-7,8$ & $0,5-1,4$ & $110-617$ & $19-56$ \\
Cereales no refinados & $1,0-2,0$ & $0,9-1,2$ & $211-618$ & $22-53$ \\
Cereales refinados & $0,5-3,2$ & $0,4-0,9$ & $30-439$ & $16-54$ \\
Pan & $0,4-0,8$ & $0,2-0,4$ & 30 & 3 \\
Tubérculos & 0,9 & 0,3 & $93-131$ & $26-31$ \\
Vegetales & $0,3-0,5$ & $0,2-0,5$ & $0-116$ & $0-42$ \\
Frutas & $0,1-0,8$ & $0,3-3,5$ & $0-63$ & $0-31$
\end{tabular}

${ }^{1}$ Adaptado de: International Zinc Nutrition Consultative Group (IZiNCG). Technical Document \#1.

Assessment of the risk of zinc deficiency in populations and options for its control. Food Nutr Bull 2004; 25(suppl 2): S94-204 
proviene de la dieta que es absorbido y utilizado por el organismo. Los factores que afectan la biodisponibilidad de zinc pueden ser tanto nutricionales como por ciertas enfermedades (p.ej. diarrea, acrodermatitis enteropática).

El fitato, componente en plantas, es un potente inhibidor de la absorción de zinc (23). El fitato consiste en la sal de magnesio, calcio, o potasio del ácido fítico (myo-inositol hexafosfato), aunque comúnmente se usa el término fitato para referirse tanto a la sal como a la molécula de ácido fítico. El mecanismo por el cual el fitato inhibe la absorción de zinc se debe a que es un poderoso quelante de minerales. Dado que el fitato no puede ser digerido o absorbido por el intestino humano, los minerales quelados al fitato también pasan por el tracto gastrointestinal sin ser absorbidos. La relación molar fitato:zinc (calculada como mg de fitato/660 dividido entre mg de zinc/65) puede ser usada para estimar la cantidad absorbible de zinc de la dieta. Adicionalmente, se ha sugerido que dietas con una relación molar fitato: zinc $>15-20$ están asociadas a una mala absorción de zinc (24). La tabla 2 muestra la cantidad total de fitato y la relación molar fitato:zinc de alimentos comúnmente consumidos por los humanos.

No se sabe con exactitud si el calcio por si solo inhibe la absorción de zinc. Varios estudios muestran que el añadir calcio a una comida o fórmula infantil no afectan negativamente la absorción de zinc (25-28), aunque existe un estudio que ha mostrado que la ingesta elevada de calcio a través de suplementos reduce la absorción neta de zinc (29). Por otro lado, la cantidad de calcio en la dieta podría afectar la absorción de zinc en condiciones donde se ingieren comidas que contienen fitato, debido a la formación de complejos entre calcio, fitato y zinc que son altamente insolubles (30).

Con respecto a hierro, pareciera que su ingesta en relaciones molares de hierro: zinc $>2.5$ inhiben la absorción de zinc cuando ambos minerales se dan como una solución acuosa (31). Sin embargo, pareciera este efecto no se duplica si ambos se ingieren junto a una comida (32). Una reciente revisión del efecto de la interacción del hierro y zinc en los parámetros bioquímicos y funcionales en intervenciones de suplementación concluyó que la evidencia no muestra un efecto adverso de la suplementación con hierro en los indicadores bioquímicos de zinc y que la suplementación conjunta de hierro y zinc tampoco parece tener un efecto negativo en zinc sérico (33). Por otro lado, también se indica que los resultados de la suplementación conjunta en niños aun no permiten sacar conclusiones firmes sobre la interacción entre el hierro y el zinc, dados conjuntamente, en el impacto sobre indicadores funcionales (33).
Finalmente, se sabe que tanto la cantidad como el tipo de proteína tienen un efecto en la absorción de zinc. A mayor cantidad de proteína, mayor la absorción de zinc, aunque cabe recalcar que la proteína es una buena fuente de zinc, por lo que a mayor ingesta de proteína mayor sería la ingesta de zinc. Respecto al tipo de proteína, la de origen animal promueve la absorción de zinc (34), mientras que se ha observado que la caseína la inhibe (25).

\section{INDICADORES DEL ESTADO DE ZINC}

Se han propuesto y utilizado, sobretodo en trabajos de investigación científica, indicadores para evaluar el estado de zinc tales como zinc en plasma o suero, en eritrocitos, en leucocitos, en pelo, en orina, actividad de ciertas enzimas dependientes de zinc (alkalina fosfatasa, ecto purina 5' nucleotidasa) y timulina sérica (32). Sin embargo, no existe a la fecha un indicador bioquímico específico y sensible para evaluar el estado de zinc de un individuo. Para la evaluación del estado de zinc de una población se recomienda el uso de zinc plasmático o sérico, pues refleja la ingesta de zinc de una población y existen valores de referencia para la mayoría de grupos de edad y de género (33).

\section{CONCLUSIONES}

El zinc es un nutriente esencial para la vida. Forma parte de numerosas enzimas en el cuerpo humano, pudiendo tener un rol catalítico, estructural o regulador. El intestino juega un rol importante en el control de la homeostasis del zinc, controlando su absorción y excreción. Buenas fuentes de zinc son alimentos de origen animal. El fitato, presente en alimentos de origen vegetal, es el mayor inhibidor de la absorción de zinc, mientras que la proteína de origen animal promueve su absorción. No existe un indicador específico y sensible para evaluar el estado de zinc de un individuo, aunque se recomienda el uso de zinc plasmático o sérico para la evaluación del estado nutricional de zinc de una población.

\section{RESUMEN}

El zinc es un catión divalente con múltiples funciones en el cuerpo humano. La absorción de zinc ocurre en el intestino delgado y es junto con la excreción de zinc vía heces, los puntos de control de la homeostasis de zinc. El zinc es un nutriente esencial con un rol específico en más de 300 enzimas, las cuales participan en todas las reacciones bioquímicas importantes del cuerpo humano. Por ende, el estado de zinc tiene un efecto directo en el crecimiento, el desarrollo neurológico y de comportamiento y en el sistema inmune. El diagnóstico de deficiencia de zinc en individuos no es posible pues 
aun no existe un marcador lo suficientemente sensible. Sin embargo, el zinc sérico es aceptable para evaluar a poblaciones. Finalmente, se pueden usar las estrategias de suplementación, fortificación y/o modificación alimentaria para controlar y/o prevenir la deficiencia de zinc.

Palabras clave: zinc; salud; homeostasis; deficiencia; intervenciones.

Dirigir la correspondencia a:

Profesor

Daniel López de Romaña

Laboratorio de Micronutrientes

Instituto de Nutrición y Tecnología de los Alimentos (INTA)

Universidad de Chile

Av. El Líbano 5524, Macul

Santiago, Chile

Teléfono: 2-9781554

Fax: 2-2214030

E-mail:dromana@inta.cl

\section{BIBLIOGRAFÍA}

1. Todd WJ, Elvehjem CA, Hart EB. Zinc in the nutrition of the rat. Am J Physiol 1934;107:146-156.

2. Prasad AS, Halsted JA, Nadimi M. Syndrome of iron deficiency anemia, hepatosplenomegaly, hypogonadism, dwarfism and geophagia. Am J Med 1961;31:532-46.

3. Vallee BL, Falchuk KH. The biochemical basis of zinc physiology. Physiol Rev 1993;73:79-118.

4. Klug A. The Discovery of Zinc Fingers and Their Applications in Gene Regulation and Genome Manipulation. Annu Rev Biochem 2010;Epub ahead of print.

5. Klug A, Schwabe JW. Protein motifs 5. Zinc fingers. FASEB J 1995;9:597-604.

6. Cousins RJ. Zinc. In: Ziegler EE, Filer LJ, eds. Present Knowledge in Nutrition, 7th ed. Washington, DC: ILSI Press, 1996:293-306.

7. Jackson MJ. Physiology of zinc: general aspects. In: Mills CF, ed. Zinc in Human Biology. London: Springer-Verlag, 1989: pp1-14.

8. Lee HH, Prasad AS, Brewer GJ, Owyang C. Zinc absorption in human small intestine. Am J Physiol 1989;256:G87-91.

9. Cousins RJ, Liuzzi JP, Lichten LA. Mammalian zinc transport, trafficking, and signals. J Biol Chem 2006;281:24085-9.

10. Lonnerdal B. Intestinal absorption of zinc. In: Mills $\mathrm{CF}$, ed. Zinc in Human Biology. London: SpringerVerlag, 1989: pp 33-55.
11. Gibson RS. Principles of Nutritional Assessment. New York-Oxford: Oxford University Press, 1990.

12. Wastney ME, Gokmen IG, Aamodt RL, Rumble WF, Gordon GE, Henkin RI. Kinetic analysis of zinc metabolism in humans after simultaneous administration of $65 \mathrm{Zn}$ and $70 \mathrm{Zn}$. Am J Physiol 1991;260:R134-41.

13. Miller LV, Krebs NF, Hambidge KM. Development of a compartmental model of human zinc metabolism: identifiability and multiple studies analyses. Am J Physiol Regul Integr Comp Physiol 2000;279:R1671-84.

14. Miller LV, Hambidge KM, Naake VL, Hong Z, Westcott JL, Fennessey PV. Size of the zinc pools that exchange rapidly with plasma zinc in humans: alternative techniques for measuring and relation to dietary zinc intake. J Nutr 1994;124:268-76.

15. Sian L, Mingyan X, Miller LV, Tong L, Krebs NF, Hambidge KM. Zinc absorption and intestinal losses of endogenous zinc in young Chinese women with marginal zinc intakes. Am J Clin Nutr 1996;63:34853.

16. Krebs NF. Overview of zinc absorption and excretion in the human gastrointestinal tract. J Nutr 2000;130:1374S-7S.

17. Istfan NW, Janghorbani M, Young VR. Absorption of stable $70 \mathrm{Zn}$ in healthy young men in relation to zinc intake. Am J Clin Nutr 1983;38:187-94.

18. Jackson MJ, Jones DA, Edwards RH, Swainbank IG, Coleman ML. Zinc homeostasis in man: studies using a new stable isotope-dilution technique. $\mathrm{Br} \mathrm{J}$ Nutr 1984;51:199-208.

19. Sian L, Hambidge KM, Westcott JL, Miller LV, Fennessey PV. Influence of a meal and incremental doses of zinc on changes in zinc absorption. Am J Clin Nutr 1993;58:533-6.

20. Weaver BP, Dufner-Beattie J, Kambe T, Andrews GK. Novel zinc-responsive post-transcriptional mechanisms reciprocally regulate expression of the mouse Slc39a4 and Slc39a5 zinc transporters (Zip4 and Zip5). Biol Chem 2007;388:1301-12.

21. Szczurek EI, Bjornsson CS, Taylor CG. Dietary zinc deficiency and repletion modulate metallothionein immunolocalization and concentration in small intestine and liver of rats. J Nutr 2001;131:2132-8.

22. Liuzzi JP, Cousins RJ. Mammalian zinc transporters. Annu Rev Nutr 2004;24:151-72.

23. O'Dell BL. Effect of dietary components upon zinc vailability. A review with original data. Am J Clin Nutr 1969;22:1315-22.

24. Turnlund JR, King JC, Keyes WR, Gong B, Michel MC. A stable isotope study of zinc absorption in 
young men: effects of phytate and alpha-cellulose. Am J Clin Nutr 1984;40:1071-7.

25. Lonnerdal B, Cederblad A, Davidsson L, Sandstrom B. The effect of individual components of soy formula and cows' milk formula on zinc bioavailability. Am J Clin Nutr 1984;40:1064-70.

26. Spencer H, Kramer L, Norris C, Osis D. Effect of calcium and phosphorus on zinc metabolism in man. Am J Clin Nutr 1984;40:1213-8.

27. Dawson-Hughes B, Seligson FH, Hughes VA. Effects of calcium carbonate and hydroxyapatite on zinc and iron retention in postmenopausal women. Am J Clin Nutr 1986;44:83-8.

28. McKenna AA, Ilich JZ, Andon MB, Wang C, Matkovic V. Zinc balance in adolescent females consuming a low- or high-calcium diet. Am J Clin Nutr 1997;65:1460-4.

29. Wood RJ, Zheng JJ. High dietary calcium intakes reduce zinc absorption and balance in humans. Am J Clin Nutr 1997;65:1803-9.

30. Lonnerdal B. Dietary factors influencing zinc ab- sorption. J Nutr 2000;130:1378S-83S.

31. Rossander-Hulten L, Brune M, Sandstrom B, Lonnerdal B, Hallberg L. Competitive inhibition of iron absorption by manganese and zinc in humans. Am J Clin Nutr 1991;54:152-6.

32. Sandstrom B, Davidsson L, Cederblad A, Lonnerdal B. Oral iron, dietary ligands and zinc absorption. J Nutr 1985;115:411-4.

33. Fischer Walker C, Kordas K, Stoltzfus RJ, Black RE. Interactive effects of iron and zinc on biochemical and functional outcomes in supplementation trials. Am J Clin Nutr 2005;82:5-12.

34. Sandstrom B, Cederblad A. Zinc absorption from composite meals. II. Influence of the main protein source. Am J Clin Nutr 1980;33:1778-83.

32. Gibson R. Assessment of chromium, copper and zinc status. In: Principles of Nutritional Assessment (2nd edition). Oxford University Press. 2005.

33. Hess S, Peerson JM, King JC and Brown KH. Use of zinc concentration as an indicator of population zinc status. Food Nutr Bull 2007; 28(3):S403-429. 